\title{
Efecto de la adición de chía sobre las características sensoriales, físico-químicas y rendimiento de la mortadela
}

\section{RESUMEN}

Los productos cárnicos cocidos tienen un déficit de fibra, el objetivo principal es investigar efectos nutricionales y económicos al sustituir fécula por harina de chía en la mortadela, obteniéndose un aporte en base seca "chía" de $23,79 \%$ proteínas, $38,47 \%$ fibra cruda, $28,96 \%$ grasa bruta, $3,28 \%$ hidratos de carbono y $5,50 \%$ cenizas, demostrando que la chía es un alimento completo y balanceado. Para la elaboración se ensayó varias dosificaciones, siendo la de mayor significancia T3 que adiciona un $5 \%$ de harina de chía, generando los siguientes resultados: $33,71 \%$ en proteínas, $5,27 \%$ en fibra cruda, $52,59 \%$ en grasa bruta, $0,02 \%$ en hidratos de carbono y $8,41 \%$ en cenizas, se destacó la fibra cruda que se incrementó desde 0,07 presente en el testigo a 5,27 en el tratamiento T3. La aceptación fue la de menor concentración por su color, por ello se adiciono $0,5 \mathrm{ml}$ de rojo carmín por kilogramo obteniendo una coloración semejante a la mortadela de las marcas locales Piggis y La Ibérica.

Palabras clave: Chía, mortadela, embutidos, fibra dietética, calidad.

EFFECT OF CHIA ADDITION ON THE SENSORY, PHYSICAL-CHEMICAL CHARACTERISTICS AND PERFORMANCE OF THE MORTADELLA

\section{ABSTRACT}

The cooked meat products have a deficit of fiber, the main objective is to investigate nutritional and economic effects when replacing starch by chia flour in the mortadella, obtaining a contribution in dry "chia" basis of $23.79 \%$ proteins, $38.47 \%$ Crude fiber, $28.96 \%$ crude fat, $3.28 \%$ carbohydrates and $5.50 \%$ ash, demonstrating that chia is a complete and balanced food. For the preparation, several dosages were tested, the most significant being $\mathrm{T} 3$, which added $5 \%$ of chia flour, generating the following results: $33.71 \%$ in proteins, $5.27 \%$ in raw fiber, $52.59 \%$ in fat Crude, $0.02 \%$ in carbohydrates and $8.41 \%$ in ash, the raw fiber that increased from 0.07 in the control to 5,27 in the T3 treatment was highlighted. The acceptance was the one of less concentration for its color, for that reason $0.5 \mathrm{ml}$ of red carmine was added per kilogram obtaining a similar coloration to the mortadela of the local brands Piggis and the Iberian.

Keywords: Chia, mortadella, sausages, dietary fiber, quality.

\section{INTRODUCCIÓN}

La industria mundial de cárnicos, con el advenimiento de la globalización y el crecimiento demográfico, ha introducido al mercado una gran variedad y cantidad de embutidos, lo que significa una millonaria cantidad de ingresos económicos a todas las personas del mundo dedicadas a esta actividad, la industria de embutidos representa un aporte valioso e importante especialmente a los países tercermundistas ya que ayuda a disminuir el hambre y la pobreza. (Ortiz, 2011)

Ecuador es un país altamente productivo que puede satisfacer las demandas alimentarias de la población, en el sector ganadero se estima una producción de 220000 toneladas métricas de carne producidas anualmente la cual se destina a consumo directo y elaboración de productos cárnicos (MAGAP, 2013).

La elaboración de productos tradicionales cocidos tales como mortadelas, salchichas, jamones, chorizos, pastel de carne, entre otros, presentan una alta proporción de lípidos saturados, colesterol e hidratos de carbono simples y son bajos en fibra e hidratos de carbono complejos (Pak, 2000).

Según la Asociación Americana de Dietética se recomienda una ingesta diaria de fibra de 25 a $35 \mathrm{~g}$ en jóvenes y adultos (Positon of the American Dietetic Association, 1996), para prevenir enfermedades crónicas, entre las que destacan el cáncer de colon, la diabetes y la hiperlipemia (OMS, 2003).

Por otra parte el mercado actual exige nuevos productos con mayor calidad, enfocando la innovación al aprovechamiento de materias primas naturales, que presenten constituyentes que incidan en la nutrición mediante el aporte simultáneo de efectos benéficos para la salud (Hillman N, 1999), que impactan al consumidor y brindan una sana alternativa de alimento en su dieta cotidiana (Ortiz, 2011).

En este contexto se encuentra el uso de la chía (Salvia hispanica L.) especie vegetal originaria del sudeste de EEUU y noroeste de América Central (México, Guatemala), cuyas cualidades nutricionales de su semilla y de los productos derivados de la misma han comenzado a ser revalorizados dado su elevado conte-

1 Docente Principal Investigador de la Escuela Superior Politécnica de Chimborazo-Ecuador E-mail: dbano@espoch.edu.ece

2 Docente de la Universidad Nacional de Chimborazo-Ecuador.

E-mail: anamejia@unach.edu.ec

3 Docente Investigador de la Universidad Nacional de Chimborazo-Ecuador. E-mail: srodas@unach.edu.ec 
nido de ácidos grasos $\omega-3$, fibra dietaria, proteínas, aminoácidos esenciales, calcio, potasio, magnesio, fosforo y antioxidantes, ofreciendo una nueva oportunidad para mejorar la nutrición humana (Jiménez, 2013).

Tomando en cuenta lo mencionado, se investigó el efecto de la adición de las semillas de chia en las características sensoriales, físico-químicas y rendimiento de la mortadela para ofrecer al mercado una mortadela con propiedades funcionales y un mejorado aporte nutricional que contribuya a mantener un buen estado de salud en la sociedad.

\section{METODOLOGÍA}

La metodología planteada en el desarrollo de la investigación se fundamentó en ensayos de laboratorio al realizar la caracterización nutricional de las semillas de chía, de la misma manera se estableció el procedimiento para la extracción de la harina integral de las semillas y se formuló mortadela sustituyendo diferentes porcentajes de la fécula por harina de chía.

Para la caracterización de las semillas de chía se realizó un análisis proximal de Weende por triplicado a tres muestras de diferentes empresas (Camari, Kunachia, Nature's gold). El el método para determinar la humedad se basó en la determinación gravimétrica de la pérdida de masa por evaporación total del agua libre y sustancias volátiles mediante el calor de la estufa a $105^{\circ} \mathrm{C}$. El método para determinar cenizas se basó en la destrucción de la materia orgánica presente en la muestra por calcinación a $550{ }^{\circ} \mathrm{C}$ y determinación gravimétrica del residuo que está constituido por óxidos, carbonatos, fosfatos y sustancias minerales. La determinación de proteína se realizó por el método Kjeldahl. La determinación de fibra se realizó por el método gravimétrico donde se somete a la muestra a una digestión ácida y alcalina obteniéndose un residuo de fibra cruda y sales que por calcinación posterior se determina la porción no digerible de la muestra. Se determinó el extracto estéreo por método Soxhlet. El extracto libre de nitrógeno se determinó por diferencia entre el cien por ciento del peso de la muestra y la suma de los porcentajes de humedad, cenizas, grasa, fibra y proteína.

Para el desarrollo de la investigación se utilizaron $36 \mathrm{~kg}$ entre carne de cerdo, carne de res, grasa de cerdo, fécula, agua y aditivos. Distribuidos en cuatro tratamientos con un peso de $3 \mathrm{~kg}$ por tratamiento (incluido el testigo) y tres repeticiones por cada uno, dando un total de 12 unidades experimentales. Los tratamientos fueron: T0 (testigo) $=$ mortadela con $0,0 \%$ de harina de chía. T1= mortadela con 1,0 $\%$ de harina de chía. T2= mortadela con 3,0 \% de harina de chía. T3= mortadela con 5,0 \% de harina de chía. La determinación de parámetros fisicoquímicos de la mortadela se realizó aplicando los métodos descritos anteriormente. Además se realizó una prueba de degustación afectiva a los cuatro tratamientos y se efectuaron pruebas de estabilidad durante treinta días a las 12 unidades experimentales, tomando en consideración parámetros microbiológicos y fisicoquímicos.

El procesamiento de datos y análisis estadístico se realizó mediante el software Microsoft Excel $($ apoyados mediante el análisis de varianza de un factor (ANOVA), la comprobación de hipótesis mediante la prueba de rangos múltiples de Duncan y la Prueba de Kruskal Wallis con un nivel de significancia de para el análisis inferencial respecto a los valores no paramétricos es decir las pruebas organolépticas. En términos generales se puede decir que la hipótesis a demostrar, dice que: H1. Mediante el uso de harina de chía en la elaboración de mortadela se mejorará la calidad nutricional y organoléptica de la mortadela.

\section{RESULTADOS Y DISCUSIONES}

\section{Valor nutricional de la mortadela \\ Proteína}

Se notó un incremento de la proteína cruda al adicionar harina de chía en los tratamientos de mortadela, siendo el mayor aporte en el tratamiento T3 con un porcentaje de $33,71 \%$.

En la Tabla 1 se reportan los resultados del análisis de varianza y de la prueba de Duncan para los datos de proteína y se observa que existe una variabilidad en la sustitución de fécula por harina de chía incrementando el aporte de proteína cruda en la mortadela. La prueba de Duncan muestra que los tratamientos 2 y 3 presentan diferencias estadísticamente significativas en comparación al testigo; es decir se demostró que al adicionar un $5 \%$ de harina de chía en la formulación de la mortadela, se obtiene un mayor valor proteico. 
Tabla 1. Análisis de la varianza y prueba de Duncan proteína en tratamientos.

\begin{tabular}{lcccc}
\hline \multicolumn{5}{c}{ Prueba de Duncan (0,05) } \\
\hline Tratamiento No. & 0 & 1 & 2 & 3 \\
RMS & & 0,223 & 0,232 & 0,237 \\
Medias & 31,39 & 31,72 & 32,49 & 33,71 \\
Dif. significativas & $\mathrm{C}$ & $\mathrm{C}$ & $\mathrm{B}$ & $\mathrm{A}$ \\
\hline
\end{tabular}

Letras iguales no existe diferencias estadísticamente significativas.

Fuente: Elaboración propia.

Los tratamientos dos y tres incrementan el valor de la proteína con lo cual cumple con la normativa vigente (NTE INEN 1338, 2012).

\section{Fibra}

En la Figura 1 Se reporta el porcentaje de fibra cruda determinada en los tratamientos y se puede evidenciar que existe variabilidad en los datos debido al porcentaje de sustitución de fécula por harina de chía incrementando el aporte de fibra cruda en la mortadela. Se aprecia también las grandes diferencias de fibra con respecto al testigo, así para el tratamiento uno hubo un incremento del $1.14 \%$, el tratamiento dos $3,67 \%$ y para el tratamiento tres 5,34 con lo cual podemos decir que el tercer producto por su cantidad de fibra llega a ser un producto funcional al incorporar un significativo aporte de fibra confiriéndole a la mortadela características nutracéuticas.

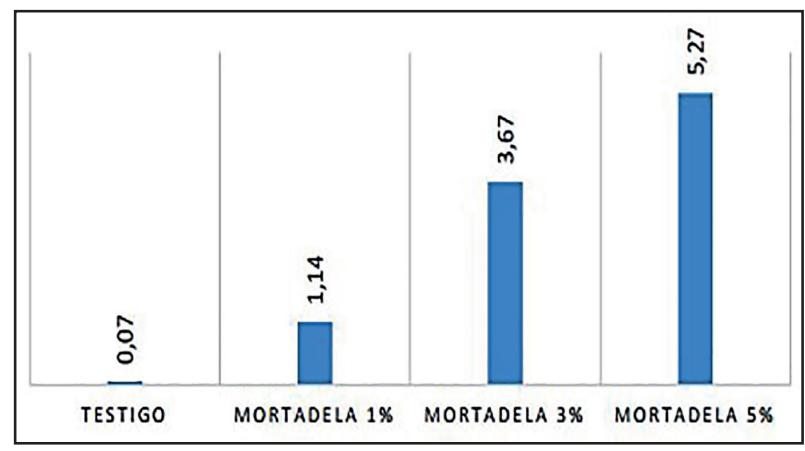

Fuente: Elaboración propia.

Figura 1. Porcentaje de fibra cruda determinada en los tratamientos.

\section{Grasa}

Se determinó un incremento de la grasa cruda al adicionar harina de chía en los tratamientos de mortadela, destacándose el mayor aporte en el tratamiento 3 (5\% de chía), siendo este del 55,59\%; Según la norma técnica para los valores nutricionales de la mortadela establece que el contenido máximo de grasa debe ser del $25 \%$, de acuerdo a los porcentajes de grasa cruda determinada en los cuatro tratamientos sobrepasan el límite máximo establecidos (NTE INEN 1340, 1996) .

\section{Análisis sensorial de los tratamientos de mor- tadela \\ Color}

Luego del análisis respectivo se determinó que si existen diferencias estadísticamente significativas entre los tratamientos como se observa en los ranks medios, esto quiere decir que la mortadela cambia su color debido a la adición de la harina de chía. (Ver Tabla 2).

Tabla 2: Prueba de Kruskal-Wallis para análisis del color en los tratamientos.

\begin{tabular}{ccccc}
\hline Tratamientos & Ranks & & \multicolumn{2}{c}{ Significancia } \\
\hline T1 & 26,83 & A & & \\
T2 & 41,58 & & B & \\
T3 & 68,08 & & & C \\
\hline
\end{tabular}

Letras iguales no existe diferencias estadísticamente significativas.

Fuente: Elaboración propia.

Además es importante considerar que en la prueba de aceptabilidad los panelistas eligen la mortadela con menor concentración de harina de chía debido a que es menos oscura que los otros tratamientos, por ello se realizaron ensayos posteriores donde se probó que se puede enmascarar el pardeamiento adicionando $0,5 \mathrm{ml}$ de rojo carmín por kilogramo de masa

\section{Palatabilidad}

Con los resultados reportados en la Tabla 3 , se determinó que solo el tratamiento T3 presenta diferencia estadísticamente significativa respecto a los tratamientos T1 y T2, esto quiere decir que la adición del $5 \%$ de chía le confiere a la mortadela características agradables, siendo aceptable para el consumidor, esto representa un importante hallazgo ya que contrario a lo que se pensaba la adición de fibra si mejoró la palatabilidad. 
Tabla 3: Prueba de Kruskal-Wallis para análisis de palatabilidad en los tratamientos.

\begin{tabular}{|c|c|l|l|l|}
\hline Tratamientos & Ranks & \multicolumn{3}{|c|}{ Significancia } \\
\hline T1 & 34,95 & A & & \\
\hline T2 & 38,52 & & A & \\
\hline T3 & 63,03 & & & B \\
\hline
\end{tabular}

Letras iguales no existe diferencias estadísticamente significativas.

Fuente: Elaboración propia.

Tabla 4: Composición química de en base seca de los tratamientos de mortadela con chía.

\begin{tabular}{|l|c|c|c|c|c|c|c|}
\hline \multicolumn{7}{|c|}{ Porcentaje en base seca } \\
\hline \multirow{2}{*}{ Muestras } & No & MS & Humedad & Cenizas & Proteínas & Lípidos & Fibra \\
\hline \multirow{4}{*}{ Mortadela 0\% } & $\mathbf{1}$ & 43,79 & 56,21 & 8,09 & 31,78 & 50,33 & 0,05 \\
\cline { 2 - 9 } & $\mathbf{2}$ & 43,97 & 56,03 & 8,05 & 31,65 & 50,25 & 0,07 \\
\cline { 2 - 9 } & $\mathbf{3}$ & 44,06 & 55,94 & 8,12 & 31,63 & 50,19 & 0,10 \\
\hline \multirow{3}{*}{$\begin{array}{l}\text { Mortadela 1\% de } \\
\text { harina de chía }\end{array}$} & $\mathbf{1}$ & 43,86 & 56,14 & 8,10 & 31,76 & 50,28 & 1,08 \\
\cline { 2 - 9 } & $\mathbf{2}$ & 44,00 & 56,00 & 8,04 & 31,69 & 50,13 & 1,22 \\
\hline \multirow{2}{*}{$\begin{array}{l}\text { Mortadela 3\% de } \\
\text { harina de chía }\end{array}$} & $\mathbf{3}$ & 43,92 & 56,08 & 8,05 & 31,72 & 50,34 & 1,12 \\
\cline { 2 - 9 } & $\mathbf{3}$ & 43,03 & 55,97 & 8,17 & 32,31 & 51,44 & 3,69 \\
\hline \multirow{2}{*}{$\begin{array}{l}\text { Mortadela 5\% de } \\
\text { harina de chía }\end{array}$} & $\mathbf{1}$ & 44,04 & 55,96 & 8,31 & 33,68 & 52,64 & 5,34 \\
\cline { 2 - 9 } & $\mathbf{2}$ & 44,09 & 55,91 & 8,50 & 33,70 & 52,55 & 5,24 \\
\cline { 2 - 9 } & $\mathbf{3}$ & 43,88 & 56,12 & 8,43 & 33,74 & 52,58 & 5,23 \\
\hline
\end{tabular}

Fuente: Elaboración propia.

En función a los resultados expresados en la Tabla 4 se puede evidenciar que en los tres tratamientos con adición de harina de chía existen variaciones del valor nutricional en relación directamente proporcional al contenido de chía que posee. Esto contrario a lo evidenciado en los estudios realizados por (Ochoa, 2014) donde menciona que la adición de harina de banano y carragenina causa el efecto contrario en lo que respecta a la grasa, proteína y fibra.

\section{CONCLUSIONES}

- La muestra de chía seleccionada para el estudio fue de la empresa Camari debido al bajo costo por kilogramo, llegando a presentar una humedad de $5,11 \%$, un aporte en base seca de $23,8 \%$ en proteína cruda, $28,9 \%$ de grasa cruda, $38,5 \%$ de fibra cruda, $5,5 \%$ de cenizas y $3,3 \%$ de extracto libre de nitrógeno.

- Al presentar un bajo porcentaje de humedad las semillas de chía pueden ser sometidas directamente al proceso de trituración para obtener harina integral, esta presenta una coloración pardo oscuro con un aroma ligeramente a pescado típico de los ácidos grasos poliinsaturados además no contiene gluten.

- Mediante el análisis de varianza se concluye que las muestras son significantemente diferentes al testigo en cuanto al contenido de fibra cruda convirtiéndose en un alimento funcional, debido a que se incrementa de un $0 \%$ a $5,3 \%$ 
en base seca al sustituir la totalidad de la fécula por harina de chía.

- Con la adición de harina de chía se mejoró el sabor y textura de la mortadela. Esto se explica ya que la chía contiene $82,8 \%$ de ácidos grasos esenciales presentes en su extracto graso, por ello se estima que del total de grasa cruda presente en la mortadela se aportaría 1,02 \% de ácidos grasos al adicionarse 5\% de harina de chía en la formulación.

- La adición de Chía en la fabricación de mortadela provocó cambio de color pero la potabilidad es aceptable.

- Respecto a la composición química de la mortadela con harina de chía se determinó una relación directamente proporcional entre la cantidad de chía adicionada con la proteína, grasa y fibra de la mortadela.

\section{REFERENCIAS BIBLIOGRÁFICAS}

[1] Hillman N, D. O. (1999). Análisis crítico de la evolución de los preparados comerciales enterales en el período de 1988 a 1996. Nutr Hosp, $14,14-22$.

[2] Jiménez, P., Masson, L., \& Quitral, V. (2013). Composición química de semillas de chía, linaza y rosa mosqueta y su aporte en ácidos grasos omega-3. Revista chilena de nutrición, 40(2), 155-160.

[3] MAGAP. (2013). Estudio de cadenas pecuarias. Ecuador.
[4] NTE INEN 1338. (2012). Obtenido de NTE INEN 1338. (2012). Carne y productos cárnicos. Productos cárnicos crudos, productos cárnicos curados-madurados, productos cárnicos precocidos-cocidos. Requisistos. Qutio, Ecuador: Norma.

[5] NTE INEN 1340. (1996). Obtenido de NTE INEN 1340. (1996). Carnes y productos cárnico: Mortadela requisitos. Quito, Ecuador: Norma.

[6] Ochoa, J. (2014). Niveles de harina de banano y carragenato semi industrial en la formulación de mortadela de pollo. Quevedo-Ecuador: Univesidad Tecnica Estatal de Quevedo.

[7] OMS. (2003). Dieta, nutrición y prevención de enfermedades crónicas. Serie de Informes Técnicos, Ginebra.

[8] Ortiz, S. (2011). Implementacionde programa de produccion mas limpia en la fabrica de embutidos catalan- Parma, para mejoramienta continuo en sus procesos y control de la contaminacion ambiental. Ambato-Ecuador: Tesis de grado Academico de Magister en produccion mas limpia UTA. FCIAL.

[9] Pak, N. (2000). La fibra dietética en la alimentación humana, importancia en la salud. In Anales de la Universidad de Chile № 11(11).

[10] Positon of the American Dietetic Association. (1996). Health implications of dietary fiber. J Am Diet Assoc ADA Reports. 
